ORIGINAL ARTICLE

\title{
A double blind, randomised, parallel group study on the efficacy and safety of treating acute lateral ankle sprain with oral hydrolytic enzymes
}

\author{
G M M J Kerkhoffs, P A A Struijs, C de Wit, V W Rahlfs, H Zwipp, C N van Dijk
}

Br J Sports Med 2004;38:431-435. doi: 10.1136/bjsm.2002.004150

See end of article for authors' affiliations

Correspondence to:

Dr Kerkhoffs, Orthopaedic Research Center

Amsterdam, Department of

Orthopaedic Surgery,

Academic Medical Center, Meibergdreef 9, PO Box

22700, Amsterdam, the

Netherlands;

ginokerkhoffs@

hotmail.com

Accepted 21 July 2003
Objective: To compare the effectiveness and safety of the triple combination Phlogenzym (rutoside, bromelain, and trypsin) with double combinations, the single substances, and placebo.

Design: Multinational, multicentre, double blind, randomised, parallel group design with eight groups structured according to a factorial design.

Setting: Orthopaedic surgery and emergency departments in 27 European hospitals.

Participants: A total of 721 patients aged 16-53 years presenting with acute unilateral sprain of the lateral ankle joint.

Primary efficacy criteria: (a) Pain on walking one or two steps, as defined by the patient on a visual analogue scale. (b) The range of motion, as measured by the investigator and expressed as a sum of flexion and extension. (c) The volume of the injured ankle measured with a volometer.

Results: At the primary end point at seven days, the greatest reduction in pain was in the bromelain/ trypsin group (73.7\%). The Phlogenzym group showed a median reduction of $60.3 \%$, and the placebo group showed a median reduction of $73.3 \%$. The largest increase in range of motion (median) was in the placebo group (60\% change from baseline). The Phlogenzym group showed a median increase of $42.9 \%$. The biggest decrease in swelling was in the trypsin group (3.9\% change from baseline). The Phlogenzym group showed a $-2.30 \%$ change from baseline and the placebo group a $-2.90 \%$ change. In the subgroup analysis of patients who did not use a Caligamed brace, Phlogenzym was superior to placebo for the summarising directional test of the primary efficacy criteria $(\mathrm{MW}=0.621 ; \mathrm{LB}-\mathrm{Cl} 0.496 ; \mathrm{p}=$ 0.029; one sided Wei-Lachin procedure). The vast majority of doctors and patients rated the tolerability of all treatments tested as very good or at least good.

Conclusions: Phlogenzym was not found to be superior to the three two-drug combinations, the three single substances, or placebo for treatment of patients with acute unilateral sprain of the lateral ankle joint. The small subgroup of patients treated without the support of a Caligamed brace showed evidence of superiority of Phlogenzym over placebo. Further research is warranted to study this effect of Phlogenzym in patients treated without ankle support.
A nkle sprains are common; one estimate is that there is one per day per 10000 of the population. ${ }^{1}$ For diagnosis of a lateral ankle ligament rupture, doctors are advised to use physical examination. ${ }^{2}{ }^{3}$ Delayed physical examination, four to seven days after the trauma, has a high sensitivity and specificity. Pain and swelling prevent an accurate diagnosis in the acute situation. Shortening the interval before diagnosis means earlier diagnosis and consequently earlier initiation of treatment. Recently, the effect of non-steroid anti-inflammatory drugs and ice on pain and swelling after an acute ankle sprain was analysed by a systematic review. Neither treatment produced a significant decrease in pain or swelling compared with no treatment. ${ }^{4}$ Proteolytic enzyme therapy is an alternative treatment for decreasing pain and swelling, used in indications such as thrombophlebitis and inflammation and in traumatic conditions. ${ }^{5}$ In ankle sprains, the enzyme may reduce swelling and pain and improve range of motion, and hence decrease the recovery time and the overall costs.

Phlogenzym (MUCOS Pharma, Geretsried, Germany) is an orally administered, anti-oedematous, anti-phlogistic drug combination. It contains three active components: the hydrolase trypsin, the endopeptidase bromelain, and the flavonoid rutoside. The drug combination is supposed to have inflammation inhibiting properties as well as a positive effect on oedema and an analgesic effect. ${ }^{6}$
The objective of this study was to assess in patients with acute lateral ankle sprain: (a) the efficacy compared with placebo treatment; $(b)$ the efficacy of the individual components and the combinations of drug compared with the efficacy of placebo and Phlogenzym; $(c)$ the safety of the triple combination Phlogenzym compared with double combinations, each substance alone, or placebo.

\section{PATIENTS AND METHODS}

Our study was a multinational, multicentre, double blind, randomised, parallel group study on the efficacy and safety of treating patients with acute lateral ankle sprain with oral hydrolytic enzymes. The study was approved by the appropriate research ethics committees. Twenty seven hospitals took part, three teaching hospitals and 24 district general hospitals, with varying catchment populations.

\section{Population}

Patients aged 16-53 years, weighing 40-160 kg, presenting to the emergency department with acute lateral ankle sprain that had occurred within the preceding 18 hours were recruited. Patients presented with pain on walking and scored the pain as $>30 \mathrm{~mm}$ on the visual analogue scale of $100 \mathrm{~mm}$. All patients suffered from a clinically swollen ankle. Table 1 lists the exclusion criteria. 


\section{Table 1 List of exclusion criteria}

1. Any injury of the ankle that required surgery.

2. Pre-existing ankle trauma.

3. History of allergy to one or more ingredients of Phlogenzym.

4. Use of immune suppressing drugs.

5. Parallel intake of non-steroidal anti-inflammatory drugs or analgesics except paracetamol only during the first two days of study treatment.

6. Known severe congenital or acquired coagulation disturbances.

7. Known renal insufficiency with serum creatinine levels $>160 \mu \mathrm{mol} / \mathrm{I}$.

8. Known severe hepatic insufficiency with either/or/and serum aspartate aminotransferase activity $>54 \mathrm{U} / \mathrm{l}$, serum alanine

aminotransferase activity $>42 \mathrm{U} / \mathrm{I}$.

9. Renal dialysis.

10. Known diabetes mellitus.

11. Known pregnancy, or lactation, or women of childbearing potential

without the use of a reliable contraceptive method.

12. Known alcohol and/or drug intoxication.

13. An operation pending which could have limited the possibility to

follow up the patient.

14. Mental disorders or mentally unable to follow exactly the trial

instructions.

15. Previously enrolled in the same trial.

16. Participation in other clinical trials with an investigational product within the 30 days before the inclusion in this study or participation more than 3 times in other clinical trials with drugs in the 12 months before inclusion.

\section{Treatments administered}

Two enteric coated tablets of Phlogenzym, placebo, trypsin (48 mg), bromelain (90 mg), rutoside ( $100 \mathrm{mg}$ ), trypsin plus bromelain, trypsin plus rutoside, or bromelain plus rutoside in the same dose were taken three times a day. This treatment was continued for 10 days. All eight test preparations were supplied by MUCOS Pharma. From day 4 until day 14 each patient had a Caligamed brace applied round the ankle (Bauerfeind Orthopaedic GmbH, Kempen, Germany).

\section{Treatment allocation}

The patients were allocated to treatment by means of a randomisation code in a random permutated block design. The test drugs were labelled numerically according to this random list. According to the list, all patients were randomly allocated to one of eight treatment groups. All test preparations were of equal size, weight, colour, smell, appearance, and taste, including the placebo.

\section{Efficacy criteria \\ Primary}

\section{Pain step test}

The pain on walking one or two steps was assessed by using a visual analogue scale with a range of $0-100 \mathrm{~mm}$.

\section{Ankle volume}

This was tested with a volometer (Boesl Medizintechnik, Aachen, Germany). This is a device to optoelectrically measure the circumference and volume of the foot. Only the volume of the ankle was measured, and measurements were expressed in plain numbers.

\section{Range of motion}

This was measured with a goniometer by the neutral zero method. Both plantar flexion and dorsal flexion of the ankle were measured and summed for total range of motion.

\section{Secondary}

The only secondary criterion was "global judgment of efficacy" assessed by the investigator.

\section{Safety criteria}

- Adverse events. The investigator recorded adverse events based on his own impression and observation.

- Global judgment of tolerability. This was assessed by both patient and investigator.

\section{Quality assurance}

A double entry of data was performed, and a standard cross check of the data was carried out, using the validated software REPORT, on a personal computer.

\section{Statistical analysis}

The three primary efficacy criteria were evaluated by the summarising Wei-Lachin procedure ${ }^{7}$ as combined criteria (generalised multivariate Wilcoxon test) on an intention to treat basis. To adjust for baseline inhomogeneities, the pain step test was evaluated as percentage change from baseline, and range of motion and volometer measurements as change from baseline (all at day 7).

With regard to the combined primary criteria, three different hypotheses were tested according to the principle of a priori ordered hypotheses: test group Phlogenzym $v$ placebo, $v$ the three two-drug groups and Phlogenzym, and the three two-drug groups combined $v$ placebo. All tests were performed as one sided tests for superiority with $\alpha=0.025$. This testing strategy allows internal validation of the study, general efficacy evaluation, and evaluation of the triple combination. The appropriate measure of relevance for the univariate Wilcoxon-Mann-Whitney tests and for the WeiLachin procedure is the Mann-Whitney estimator $(\mathrm{MW}){ }^{8}$ Data were evaluated using the validated software SMARTEST.

\section{RESULTS}

\section{Study population}

Overall, 721 patients were randomised between June 1999 and August 2000. Twenty nine were excluded because they received no or no certain treatment; a further 18 were excluded because they committed severe protocol violations. A total of 674 patients were analysed on an intention to treat basis. Figure 1 shows the flow of patients through the trial.

\section{Baseline characteristics}

The baseline characteristics of patients in all eight groups were well balanced (table 2). With regard to the summarised baseline efficacy criteria, the combined Mann-Whitney estimators (Wei-Lachin) are situated between $\mathrm{MW}=0.44$ and $\mathrm{MW}=0.56$, indicating only small differences. However, an additional comparison of the combined active treatment groups with placebo shows significant superiority of the active treatments at baseline, indicating that the active treatment groups contained less affected patients $(\mathrm{p}=$ 0.039; two sided). Therefore, so called "floor effects" may reduce the test power of the final efficacy evaluation.

\section{Efficacy}

Pain step test

Starting with baseline visual analogue scale scores of 60.463.5 (means), all groups showed a considerable decrease in pain on day 7 (primary end point) with means of 20.5-26.8, and a further decrease on day 14 with final means of 10.715.0. The largest decrease was observed in the first four days. Figure 2 shows the time course of the absolute values (Phlogenzym, two-drug combinations, placebo).

At day 7, the largest median reduction in pain was in the bromelain-trypsin group $(73.7 \%)$. The Phlogenzym group showed a median reduction of $60.3 \%$, and the placebo group a median reduction of $73.3 \%$. 

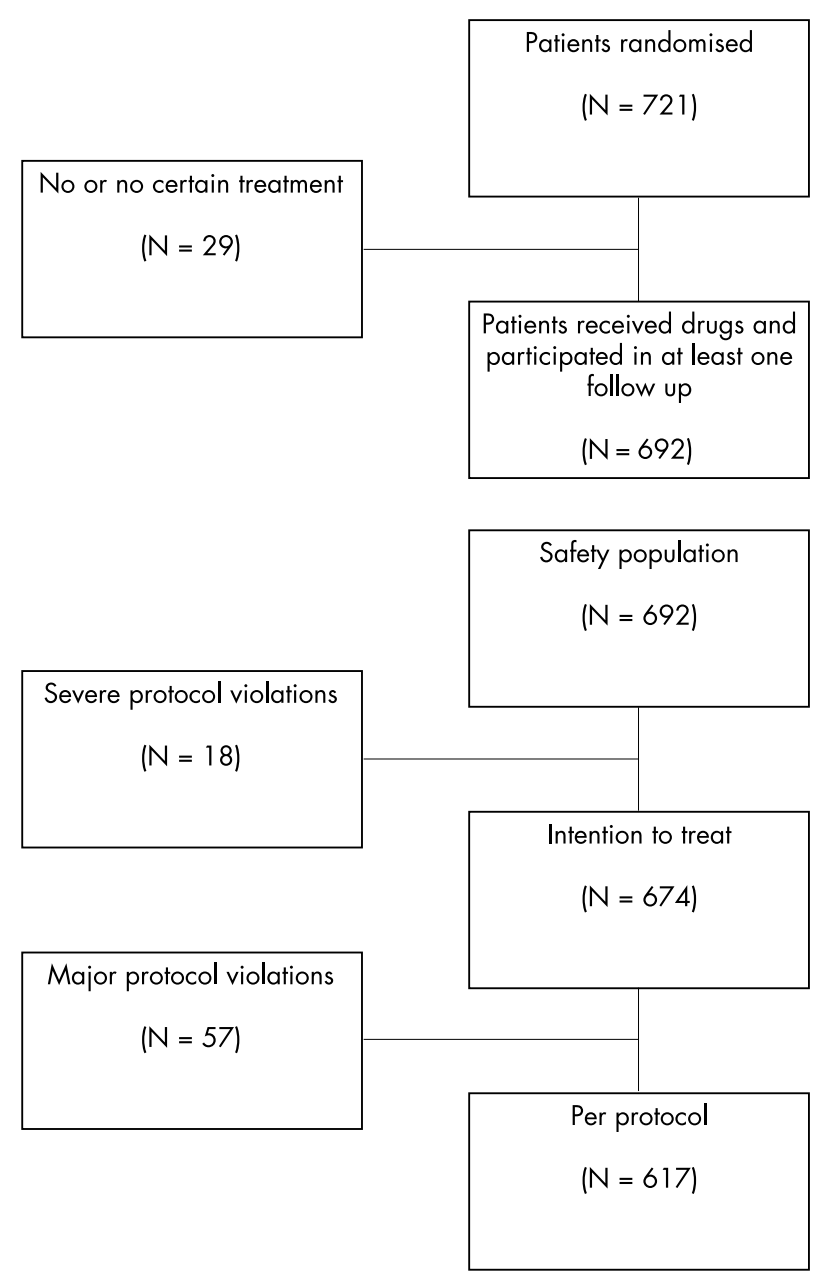

Figure 1 Progress of participants through the trial.

\section{Ankle volume}

Starting with baseline volometer measurements of the injured foot of $2540-2610$ (means), all groups showed a decrease in volume at day 7 , with means of 2453-2524, and a further decrease at day 14, with final means of 2420-2486. Figure 3 shows the time course of the changes from baseline (Phlogenzym, two-drug combinations, placebo).

The biggest decrease in swelling after seven days of treatment with regard to the means was in the trypsin group (change from baseline -105.3), and the smallest decrease

Table 2 Baseline values for outcome measures of treatment of acute ankle injury

\begin{tabular}{llll}
\hline \multicolumn{4}{l}{ Outcome measure } \\
\cline { 2 - 4 } Group & Pain & Range of motion & Swelling \\
\hline Phlog & $60.5(18.0)$ & $31.7(13.1)$ & $2559(408)$ \\
$\mathrm{Br}-\mathrm{Tr}$ & $60.4(18.1)$ & $31.1(11.3)$ & $2586(379)$ \\
$\mathrm{Br}-\mathrm{Ru}$ & $63.4(17.3)$ & $32.7(13.3)$ & $2589(376)$ \\
$\mathrm{Br}$ & $62.8(19.7)$ & $31.1(12.8)$ & $2540(413)$ \\
$\mathrm{Tr}-\mathrm{Ru}$ & $61.7(18.1)$ & $33.2(13.5)$ & $2445(299)$ \\
$\mathrm{Tr}$ & $61.5(16.1)$ & $33.2(15.6)$ & $2579(390)$ \\
$\mathrm{Ru}$ & $63.5(17.8)$ & $32.9(12.6)$ & $2550(409)$ \\
Placebo & $63.4(19.9)$ & $29.8(10.7)$ & $2610(360)$ \\
\hline
\end{tabular}

Values are mean (SD). Pain is measured on a visual analogue scale (1100). Range of motion is measured with a goniometer, expressed as the sum of dorsal and plantar flexion in degrees. Swelling is measured using a volometer, expressed in plain numbers, not $\mathrm{mm}^{3}$.

Phlog, Phlogenzym; Br, bromelain; Tr, trypsin; Ru, rutoside.

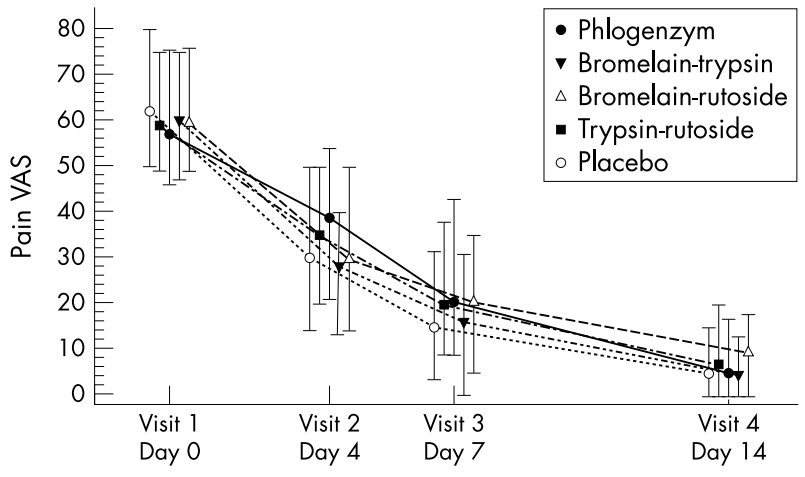

Figure 2 Effect of Phlogenzym, drug combinations, and placebo on pain of ankle sprain measured on a visual analogue scale (VAS). Absolute values (medians, lower and upper quartiles) are shown. Data set: intention to treat. Missing values replaced by last value carried forward (LVCF).

was in the trypsin-rutoside group (change from baseline -60.5). The Phlogenzym group showed a mean decrease of 76.1 , and the placebo group showed a mean decrease of 85.8 .

\section{Range of motion}

Starting with baseline ranges of motion of 29.8-33.2 (means), all groups showed a considerable increase in range of motion at day 7 , with means of $43.6-48.9^{\circ}$. The largest was observed within the first 4 days. Figure 4 shows the time course of the absolute values (Phlogenzym, two-drug combinations, placebo).

The largest mean increase in range of motion after seven days of treatment was in the placebo group $\left(+17.1^{\circ}\right.$ change from baseline) and the smallest increase was in the Phlogenzym group $\left(+12.0^{\circ}\right.$ change from baseline $)$. At the end of the study (visit 4) the largest increase was in the placebo group $\left(+23.8^{\circ}\right.$ mean change from baseline), whereas the smallest increase was observed in the trypsin-rutoside group $\left(+18.4^{\circ}\right.$ mean change from baseline). The Phlogenzym group showed a final change from baseline of $+18.7^{\circ}$ (table 3 ).

\section{Test results primary efficacy criteria}

Hypothesis 1: Phlogenzym group $v$ placebo

The Phlogenzym group showed some inferiority (odds ratio 0.4-0.7) with regard to the combined efficacy criteria (MW $=0.406)$. The difference is not significant $(\mathrm{p}=0.996$; one

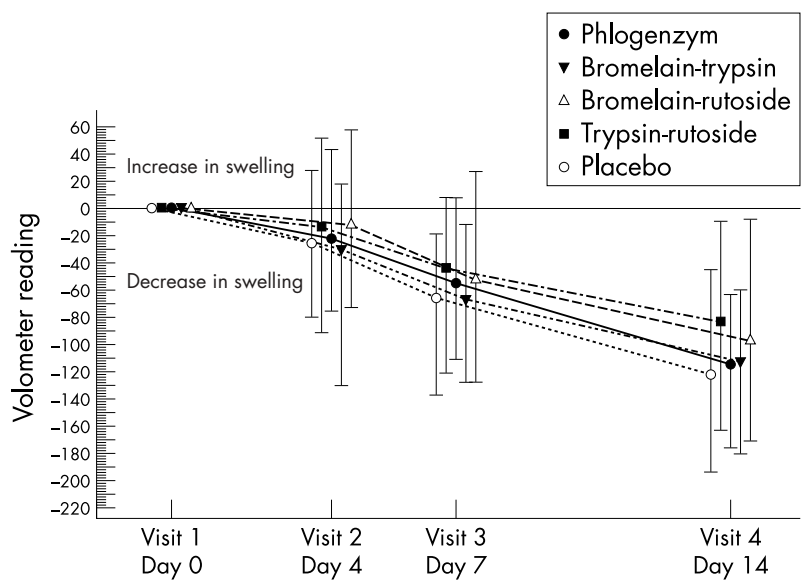

Figure 3 Effect of Phlogenzym, drug combinations, and placebo on volume of ankle measured by volometer. Change from baseline (medians, lower and upper quartiles) is shown. Data set: intention to treat. Missing values replaced by last value carried forward (LVCF). 


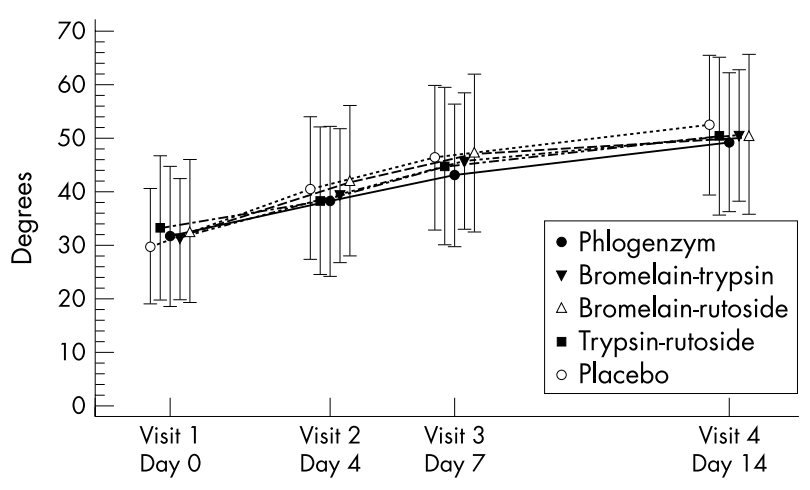

Figure 4 Effect of Phlogenzym, drug combinations, and placebo on range of motion after ankle sprain (degrees of flexion and extension). Absolute values (means and standard deviations) are shown. Data set: intention to treat. Missing values replaced by last value carried forward (LVCF).

sided Wei-Lachin procedure). As this test for hypothesis 1 is not significant, we interpreted the other tests in an exploratory manner.

Hypothesis 2: Phlogenzym group $v$ two-drug groups Phlogenzym could not be shown to be superior to the three two-drug groups in the predefined sense (Phlogenzym $v$ the combined three two-drug groups: $\mathrm{MW}=0.467 ; \mathrm{p}=0.922$; one sided Wei-Lachin tests for superiority; exploratory interpretation). The Mann-Whitney estimator indicates that Phlogenzym is slightly inferior in efficacy.

\section{Hypothesis 3: Phlogenzym and two-drug groups $v$ placebo}

The Phlogenzym group and the three two-drug combinations together were slightly inferior with regard to the combined efficacy criteria $(\mathrm{MW}=0.446 ; \mathrm{p}=0.995$; one sided WeiLachin procedure; exploratory interpretation).

\section{Secondary efficacy criterion: investigator assessment global judgment of efficacy}

Therapeutic success (judgment "good" or "very good") was achieved by different proportions of patients in the different treatment groups. The best result was found in the bromelain-trypsin group (78.7\%). In the Phlogenzym group, $78.5 \%$ of the cases were judged as good or very good. On the secondary criterion, global assessment of efficacy by the investigator, Phlogenzym was found to be slightly superior to placebo in the full analysis set, with $\mathrm{p}=0.049$ ( $\mathrm{MW}=$ 0.5721 , Wilcoxon-Mann-Whitney test; one sided Wei-Lachin procedure; intention to treat; exploratory interpretation).

\section{Subgroup analysis}

\section{Caligamed brace}

A small proportion of patients in each group refused to wear a Caligamed brace: $7.0 \%$ in the Phlogenzym group and $4.8 \%$ in the placebo group. A supportive analysis of the primary efficacy criteria was performed for this subgroup of patients. The combined Mann-Whitney estimators indicate that Phlogenzym is slightly more efficacious than placebo (MW $=0.6213$, lower border confidence interval (LB-CI) 0.4958; $\mathrm{p}=0.029$; one sided Wei-Lachin procedure). To validate these results, we performed a further supportive analysis using global judgment of efficacy by the investigator. This test revealed a great superiority of Phlogenzym $(\mathrm{MW}=$ 0.8250; LB-CI 0.5469; p = 0.0873; one sided Wei-Lachin procedure).

\section{Safety}

Adverse events

A total of 124 adverse events were recorded. The placebo group had the fewest (11/12.8\%), and the Phlogenzym group the most $(24 / 26.7 \%)$. Comparing the intensities of the adverse events and the judgments in relation to treatment, no substantial differences between the Phlogenzym and placebo groups were found. Also no strikingly different patterns were identified between placebo and the single components of Phlogenzym and their additionally tested combinations. There was only one serious adverse event in this study: one patient developed a thrombosis of the right calf. The patient discontinued the study and after 12 days had recovered completely. The investigator considered this event to be related to the underlying study indication (and thus not related to the test treatment). Out of 692 patients in the safety population, $13(1.9 \%)$ discontinued the study because of adverse events.

\section{Global judgment of tolerability}

In all treatment groups, the vast majority of doctors and patients rated the tolerability of the test preparations as good or very good. Equivalence in a descriptive sense was demonstrated between Phlogenzym and placebo $(\mathrm{MW}=$ 0.5191; CI-LB 0.4411). There were no substantial differences between the other groups and placebo, the judgments of the doctors and patients showing a large degree of agreement.

\section{DISCUSSION}

The aim of this study was to determine the contribution of individual components to the effect of the combination drug in comparison with placebo, and to compare the efficacy of the combination of Phlogenzym with the individual substances and placebo treatment. Although some clinical trials ${ }^{9}{ }^{10}$ have indicated that Phlogenzym is effective in the treatment of lateral ankle ligament injury, we could not show

Table 3 Results of the different treatments of acute ankle injury

\begin{tabular}{|c|c|c|c|c|c|c|c|c|c|}
\hline \multirow[b]{2}{*}{ Group } & \multicolumn{3}{|c|}{ Decrease in pain } & \multicolumn{3}{|c|}{ Range of motion } & \multicolumn{3}{|c|}{ Decrease in swelling } \\
\hline & Day 2 & Day 7 & Day 14 & Day 2 & Day 7 & Day 14 & Day 2 & Day 7 & Day 14 \\
\hline Phlog & $23.0(18.6)$ & $34.3(22.7)$ & 49.1 (19.3) & $6.8(10.1)$ & $12.0(11.5)$ & $18.7(14.4)$ & $25.6(200)$ & 76.1 (215) & 139.1 (199) \\
\hline $\mathrm{Br}-\mathrm{Tr}$ & $31.0(19.2)$ & $39.7(22.4)$ & 49.7 (18.9) & $8.5(10.1)$ & 15.0 (13.5) & $20.6(12.8)$ & 48.0 (187) & 79.4 (177) & $142.3(179)$ \\
\hline $\mathrm{Br}-\mathrm{Ru}$ & $30.1(20.8)$ & $40.1(21.4)$ & $51.4(19.8)$ & $9.7(10.6)$ & 15.1 (13.5) & $19.3(14.1)$ & 23.6 (175) & 80.9 (214) & 105.7 (184) \\
\hline $\mathrm{Br}$ & $27.0(22.9)$ & $36.0(23.5)$ & $48.0(22.1)$ & $10.0(12.1)$ & $14.8(12.7)$ & $19.9(14.2)$ & $41.5(140)$ & $87.8(201)$ & 115.9 (174) \\
\hline Tr-Ru & $25.1(20.8)$ & 36.2 (21.9) & $46.8(19.4)$ & $5.5(11.7)$ & $12.2(13.8)$ & $18.4(15.1)$ & 12.8 (137) & 60.5 (125) & 90.9 (125) \\
\hline $\operatorname{Tr}$ & $26.1(18.9)$ & $38.2(20.5)$ & 46.5 (19.7) & 8.1 (13.5) & 14.6 (15.5) & $19.8(16.4)$ & 61.2 (149) & $105.3(151)$ & $124.4(152)$ \\
\hline Ru & $27.7(22.1)$ & $37.7(20.7)$ & $50.5(20.2)$ & $9.4(11.7)$ & $16.0(11.1)$ & $21.0(14.4)$ & 27.2 (123) & $73.6(123)$ & 104.3 (115) \\
\hline Placebo & $30.8(20.1)$ & $43.0(21.4)$ & $52.8(21.0)$ & $11.1(12.2)$ & $17.1(12.7)$ & 23.8 (13.0) & 35.0 (121) & 85.8 (132) & 123.5 (132) \\
\hline
\end{tabular}

Values are mean (SD). Pain is measured on a visual analogue scale (1-100). Range of motion is measured with a goniometer, expressed as the sum of dorsal and plantar flexion in degrees. Swelling is measured using a volometer, expressed in plain numbers, not $\mathrm{mm}^{3}$.

Phlog, Phlogenzym; Br, bromelain; Tr, trypsin; Ru, rutoside. 


\section{Take home message}

Administration of proteolytic enzymes is no more effective than placebo in patients with an acute lateral ankle sprain treated functionally with a brace.

any benefit over placebo or the three two-drug combinations. Various parametric and non-parametric simple and factorial analyses revealed no additional benefit of the three single substances bromelain, trypsin, and rutoside, nor relevant interactions. The active treatment groups had considerably lower baseline levels than the placebo group $(p=0.039$; two sided Wei-Lachin procedure). With lower baseline disease activity, "floor effects" may have meant that the study treatment had no chance of showing superiority. Although the primary efficacy criteria failed to confirm the superiority of Phlogenzym, the secondary criterion, global assessment of efficacy by the investigator, shows a slight superiority of Phlogenzym over placebo in the full analysis set $(\mathrm{p}=0.049$; one sided Wei-Lachin procedure). The global assessment was the investigator's opinion of the overall efficacy of the study treatment in controlling swelling and pain. This positive outcome is probably influenced by the difference in baseline levels: assessment was likely to be positive if symptoms were more obvious at inclusion.

Another reason for the failure to demonstrate the efficacy of Phlogenzym may be that the clinical model was not suitable and the efficacy criteria were not responsive enough. A number of studies with Phlogenzym ${ }^{5}{ }^{6}$ have shown that the full treatment effect of enzymes only develops after a certain latency period of, possibly, more than a week. In the model of acute ankle sprain, primary traumatic and possibly only secondary inflammatory symptoms were present. Enzyme treatment was started about 18 hours after the trauma and continued for 10 days. By this time, bleeding has usually stopped and secondary oedema has reached its peak. Reparative reaction can be expected to have already started at the start of the enzyme treatment. The number of patients who accepted the Caligamed brace as a primary treatment was high. However, some patients refused the use of an ankle support for unknown reasons. These patients had comparable baseline characteristics. In the group treated without support, there is strong evidence for the efficacy of Phlogenzym. The use of a brace can be assumed to result in a faster return to normal range gate and range of motion of the ankle. It seems therefore that the enzyme is more efficient in combination with movement than with immobilisation. This theory, however, should be further analysed.

Another possible source of bias was the fact that no intraobserver and interobserver testing was performed on the outcome measure range of motion. However, the chance of bias was maximally reduced by using a standardised measurement method.

The total number of eligible patients presenting to the participating emergency departments during the recruitment period was not accurately noted because of the busy nature of the setting. Probably a minority of eligible patients were recruited because recruitment depended on the motivation of local staff. Nevertheless, the results are widely applicable because the reasons for not recruiting patients-for example, department too busy, shortage of staff, lack of motivation of staff on duty-are unlikely to be related to the characteristics of the patients. The fact that the study was carried out in several hospitals also supports the applicability of the findings. No other potential sources of bias could be identified.

Considering all results of the safety analyses, the tolerability of Phlogenzym may be rated as very good. However, as this study failed to demonstrate its efficacy, proteolytic enzyme treatment with Phlogenzym clearly cannot yet provide the solution for shortening the delay in diagnosis of an acute lateral ankle ligament injury.

Further research is warranted to compare the effect of Phlogenzym in patients treated with and without an ankle support.

\section{CONCLUSIONS}

- In patients with an acute ankle sprain, treatment with Phlogenzym could not be clearly shown to be superior to the three two-drug combinations, the three single substances, or even placebo.

- The tolerability of Phlogenzym can be rated as very good.

- Proteolytic enzyme treatment with Phlogenzym does not yet clearly shorten the delay in diagnosis of an acute lateral ankle ligament injury.

\section{Authors' affiliations}

G M M J Kerkhoffs, P A A Struijs, C de Wit, C N van Dijk, Orthopaedic Research Center Amsterdam, Department of Orthopaedic Surgery, Academic Medical Center, University of Amsterdam, the Netherlands V W Rahlfs, Data Analysis and Study Planning, IDV, Wessobrunnerstrasse 6, D-82131 Gauting, Germany

H Zwipp, Unfall- und Wiederherstellungschirurgie, Technische Universitaet Dresden, Germany

\section{REFERENCES}

1 Katcherian D. Soft-tissue injuries of the ankle. In: Lutter LD, Mizel MS, Pfeffer GB, eds. Orthopaedic knowledge update: foot and ankle. Rosemont, IL: American Academy of Orthopaedic Surgeons, 1994:241-53.

2 Van Dijk CN, Lim LSL, Bossuyt PMM, et al. Physical examination is sufficient for the diagnosis of sprained ankles. J Bone Joint Surg $[\mathrm{Br}]$ 1996;78:958-62.

3 Klenerman $L$. The management of sprained ankle [editorial]. J Bone Joint Surg [Br] 1998:80:11-12.

4 Van Dijk CN. [CBO-guideline for diagnosis and treatment of the acute ankle injury. National organization for quality assurance in hospitals.] (In Dutch.) Ned Tijdschr Geneeskd 1999;143:2097-101.

5 Simerda P. 52-week toxicology study on dogs. Final report, MUCOS Pharma, Geretsried, data on file, 1994.

6 Hoernecke R, Doenicke A. Peri-operative enzyme therapy: a useful complement to the post-operative pain treatment. Anaesthesist 1993;42:856-61.

7 Wei L, Lachin JM. Two sample asymptomatically distribution-free tests for incomplete multivariate observations. J Am Stat Assoc 1984;79:653-61.

8 Colditz GA, Miller JN, Mosteller F. Measuring gain in the evaluation of medical technology: the probability of a better outcome. Int J Technol Assess Health Care 1988;4:637-42.

9 Hollmann W. Efficacy and safety of hydrolytic enzymes and rutin in patients with distortions of the ankle joint. Clinical study report MU-694411, idvDatenanalyse und Versuchsplanung Gauting, 1998.

10 Van Dijk CN. A double blind comparative study on the efficacy of MU-410 vs. placebo in patients with acute disruption of the anterior fibulotalar ligament. Study Nr. 4903XV, Amsterdam, 1994. 